$\mathrm{CO}_{2}$ Capture Performance Comparisons of Polyamines at Practical Concentrations for Use as Activators for MDEA for Natural Gas Sweetening

Bander Jaffary, Laila Jaafari and Raphael Idem*

Clean Energy Technologies Research Institute (CETRI), Faculty of Engineering \& Applied Science, University of Regina, 3737 Wascana Parkway, Regina, Saskatchewan S4S 0A2, Canada Supporting Information

Table S1. Kinetic results of absorption process using screening data of single polyamines with high amine concentration.

\begin{tabular}{|l|cc|cc|}
\hline & \multicolumn{2}{|c|}{ With respect to $\mathrm{CO}_{2}$} & \multicolumn{2}{l|}{ With respect to amine } \\
\hline System & $\mathrm{n}$ & $\mathrm{k}$ & $\mathrm{m}$ & $\mathrm{k}$ \\
\hline PZ & 0.96 & 0.1916 & 0.65 & 0.0777 \\
TEPA & 0.92 & 0.1616 & 0.39 & 0.0658 \\
PEI-B & 0.61 & 0.1268 & 0.12 & 0.0738 \\
\hline
\end{tabular}

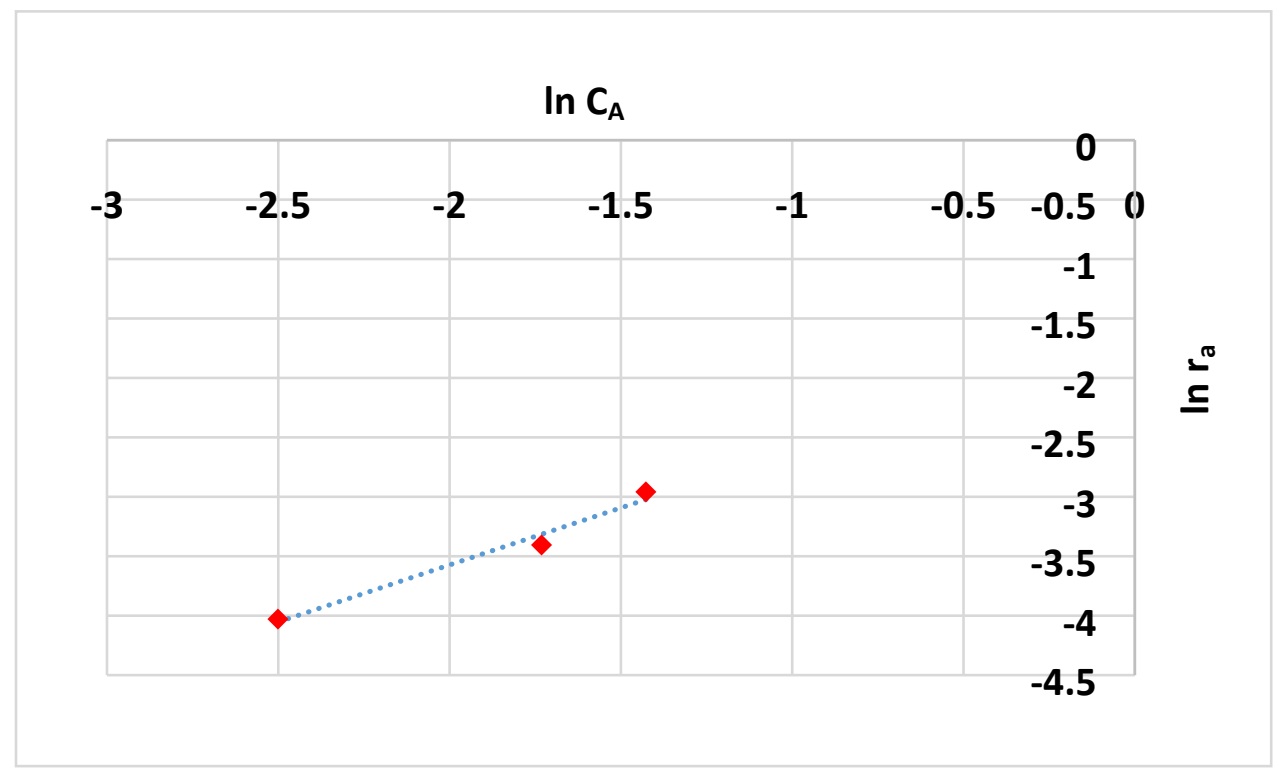

Figure S1. Natural logarithm of $\mathrm{CO}_{2}$ concentration versus natural logarithm of $\mathrm{CO}_{2}$ absorption rate for PZ based on pseudo power law model. 


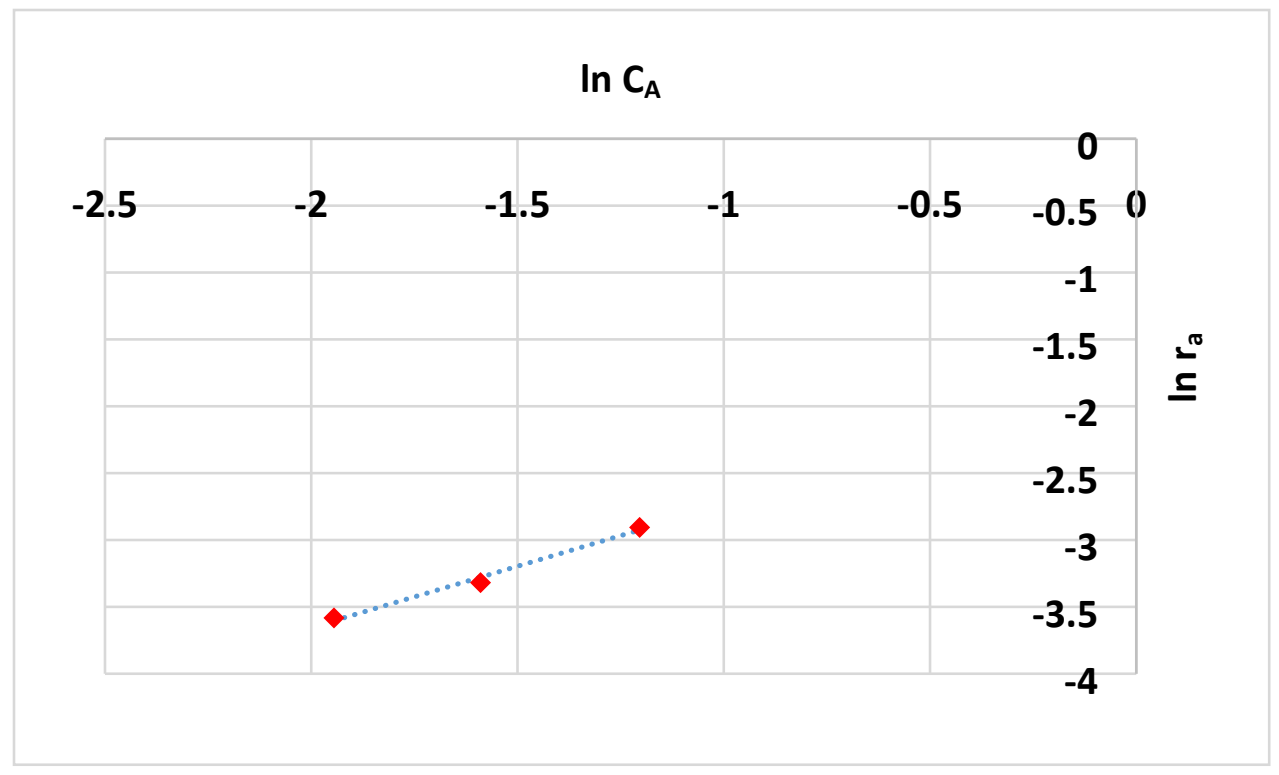

Figure S2. Natural logarithm of $\mathrm{CO}_{2}$ concentration versus natural logarithm of $\mathrm{CO}_{2}$ absorption rate for TEPA based on pseudo power law model.

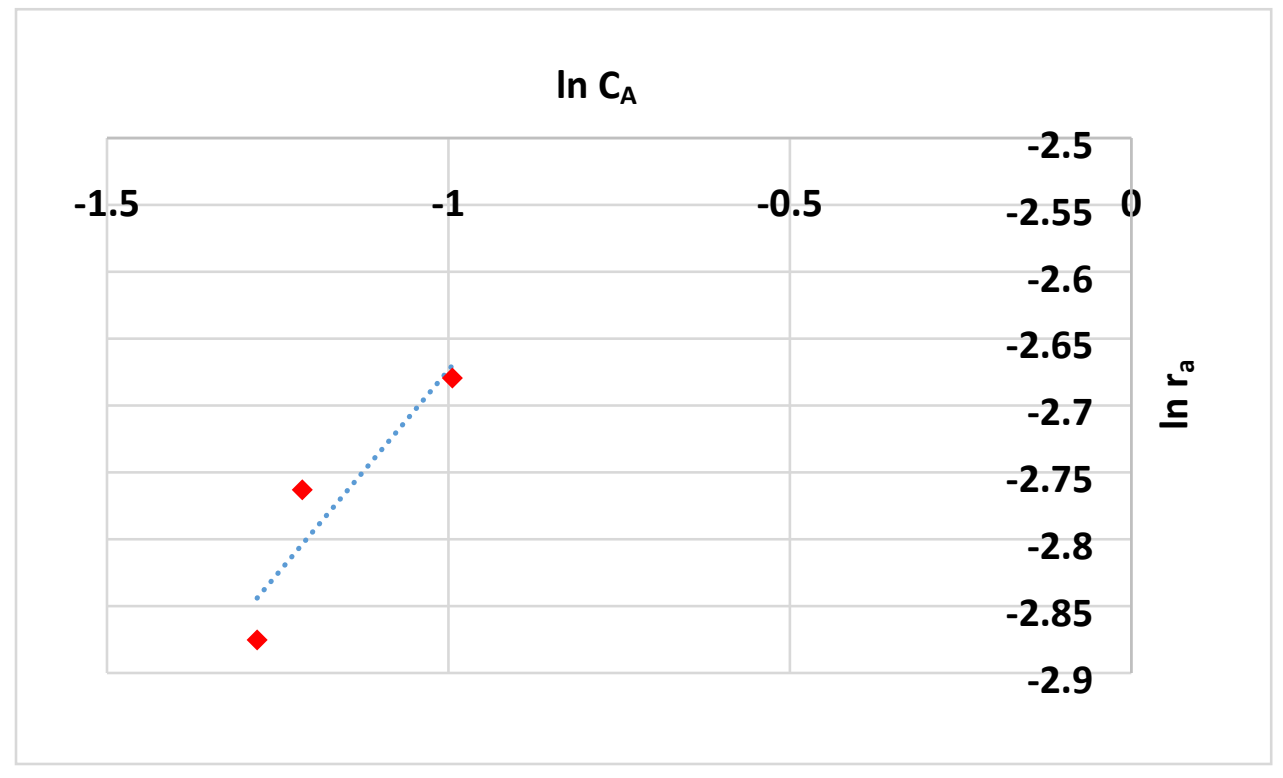

Figure S3. Natural logarithm of $\mathrm{CO}_{2}$ concentration versus natural logarithm of $\mathrm{CO}_{2}$ absorption rate for PEI-B based on pseudo power law model. 


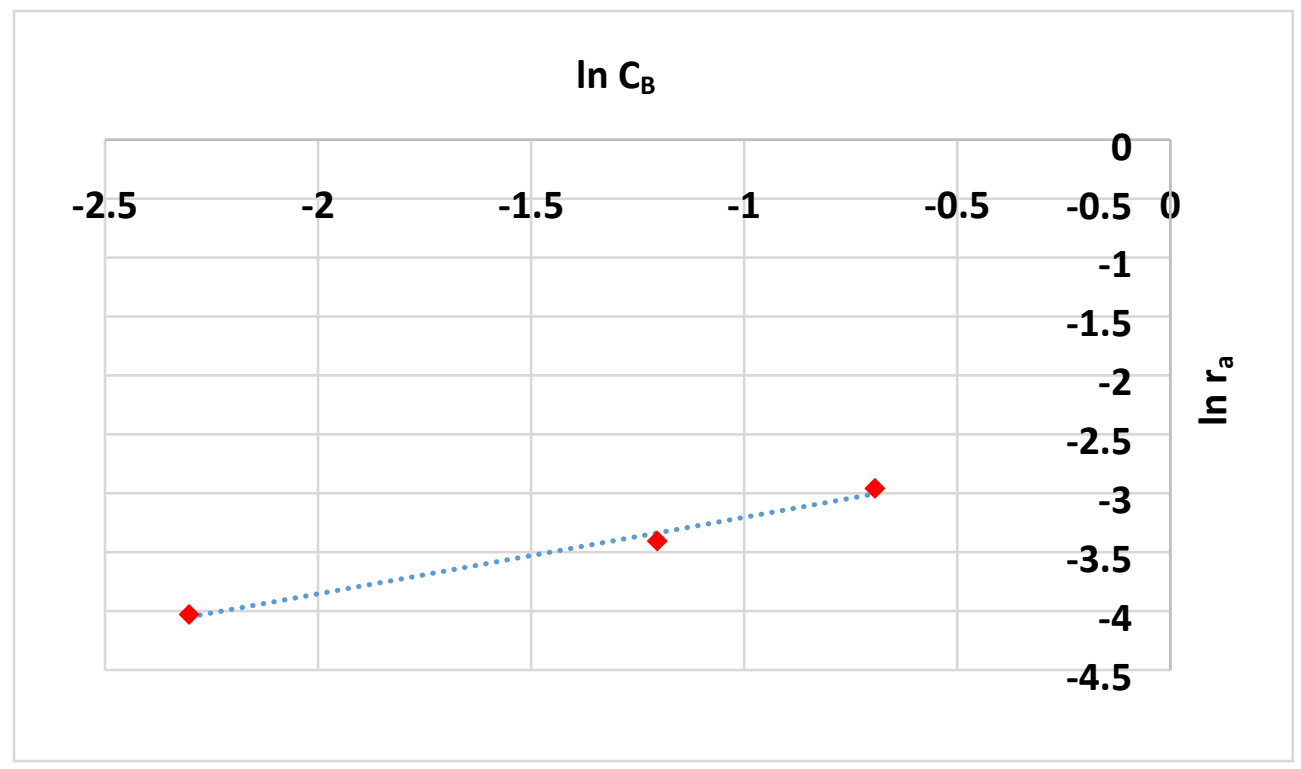

Figure S4. Natural logarithm of amine concentration versus natural logarithm of $\mathrm{CO}_{2}$ absorption rate for PZ based on pseudo power law model.

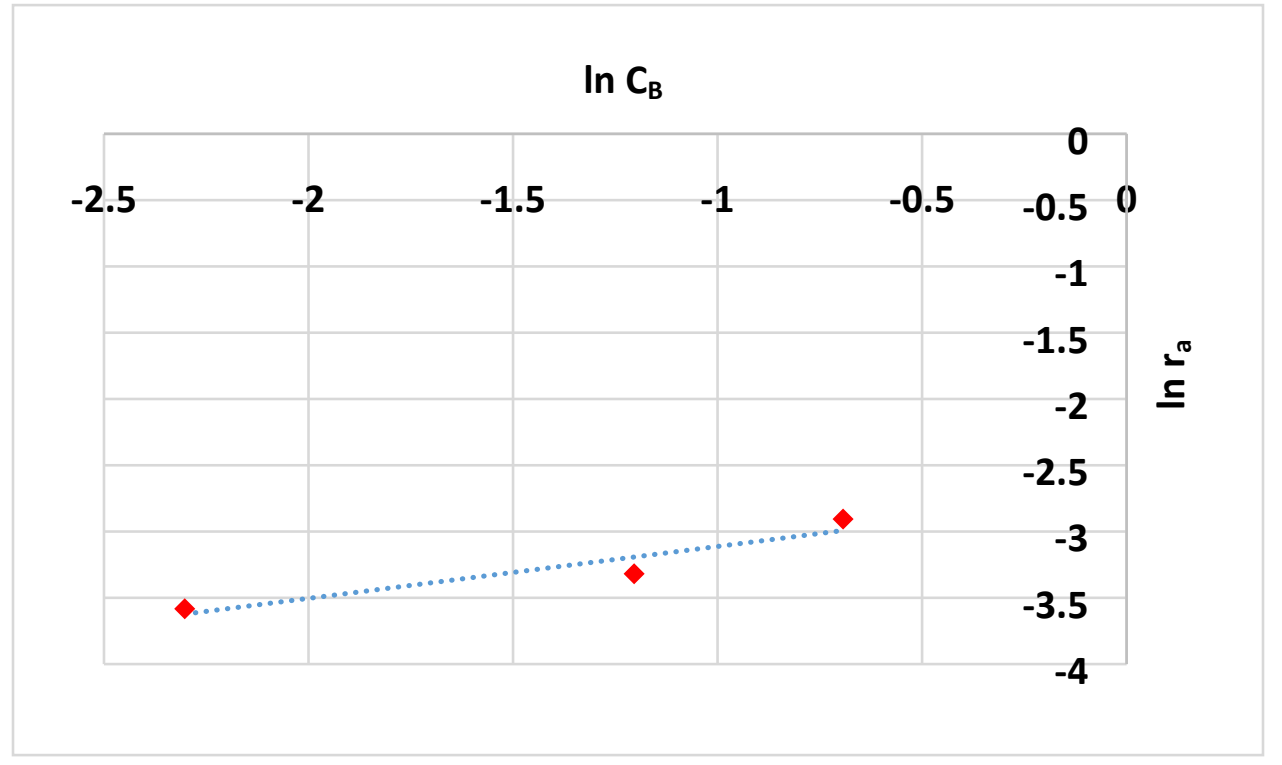

Figure S5. Natural logarithm of amine concentration versus natural logarithm of $\mathrm{CO}_{2}$ absorption rate for TEPA based on pseudo power law model. 


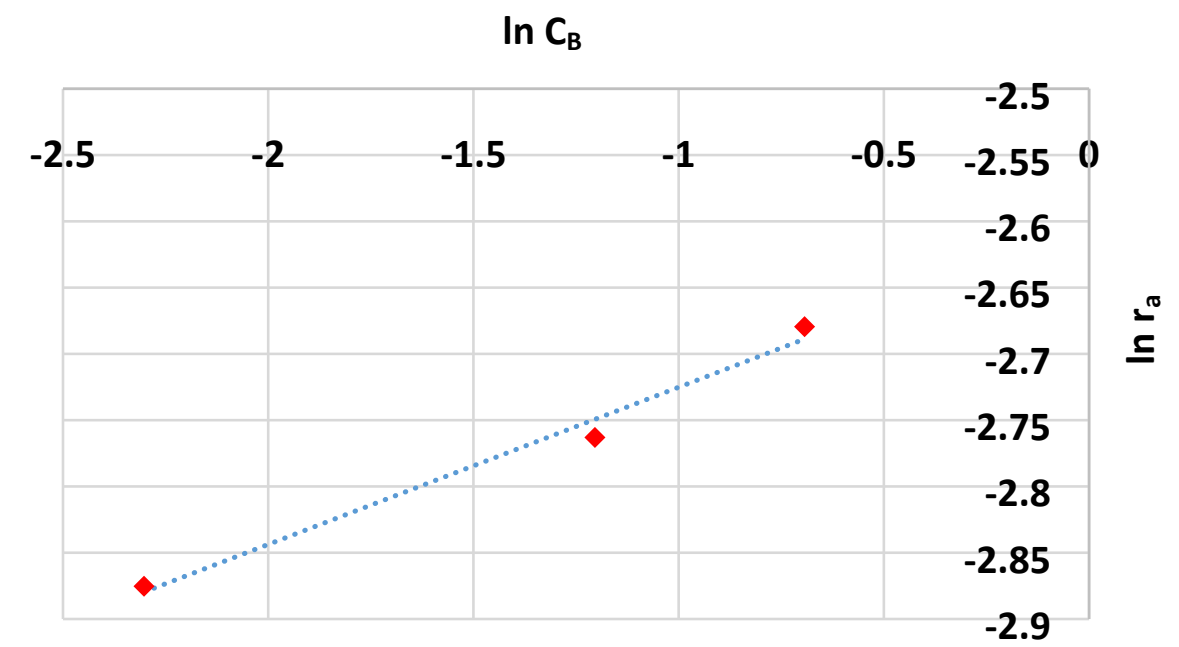

Figure S6. Natural logarithm of amine concentration versus natural logarithm of $\mathrm{CO}_{2}$ absorption rate for PEI-B based on pseudo power law model.

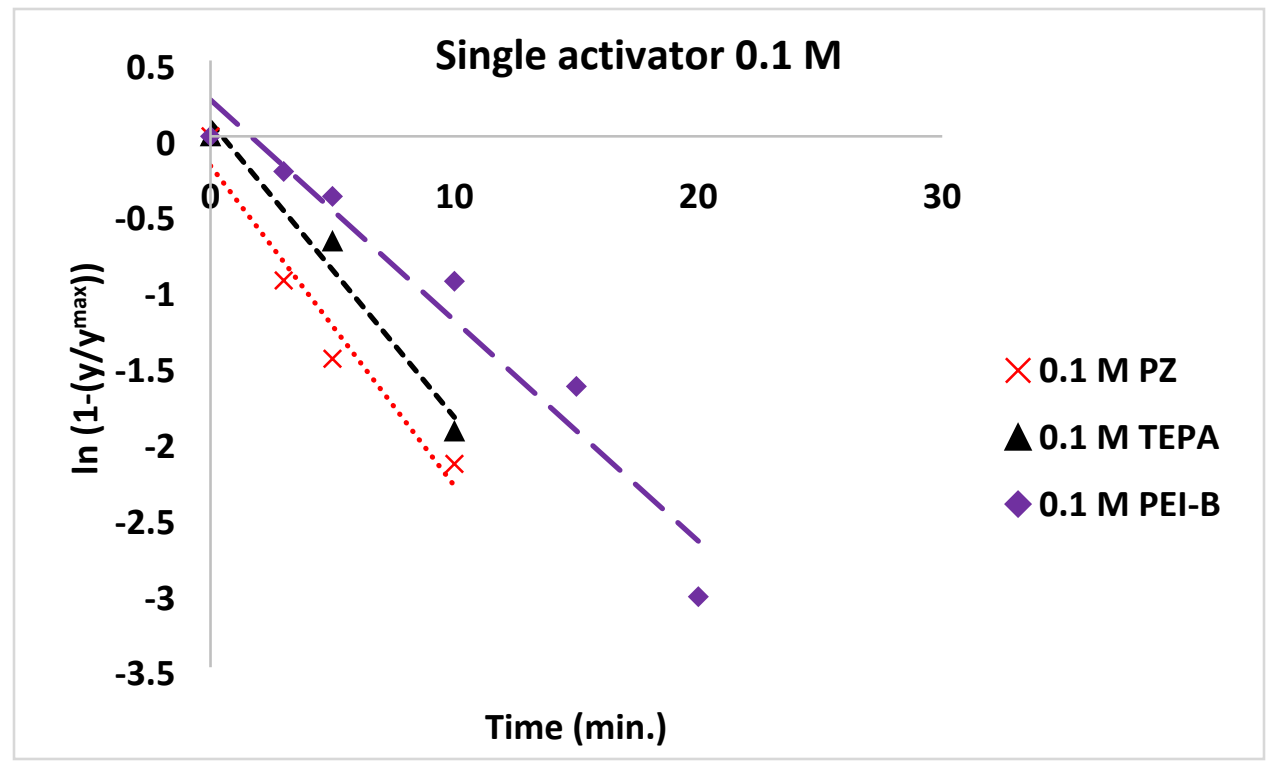

Figure S7. Plot of first order exponential rise expression using $0.1 \mathrm{M}$ polyamine concentration. 


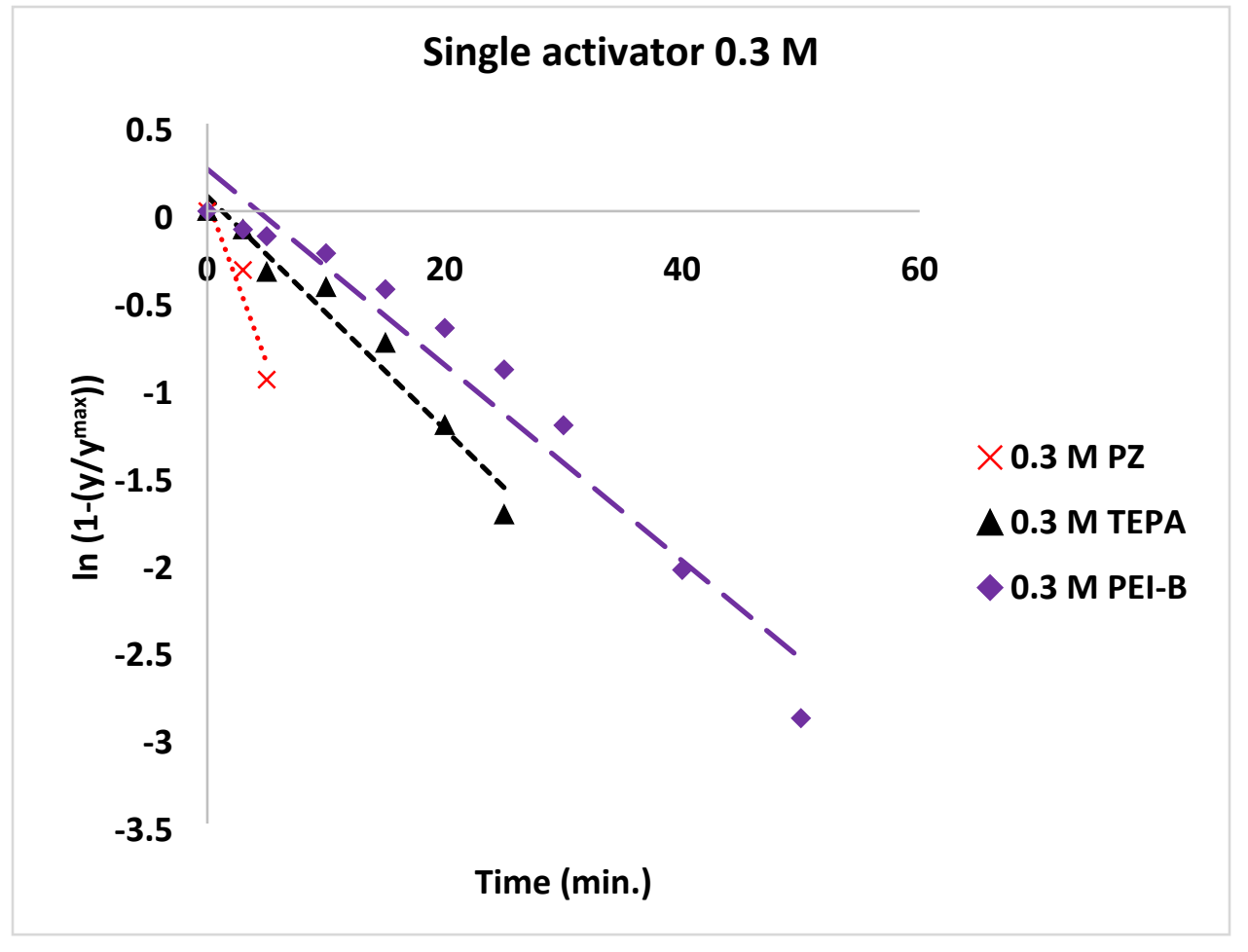

Figure S8. Plot of first order exponential rise expression using $0.3 \mathrm{M}$ polyamine concentration.

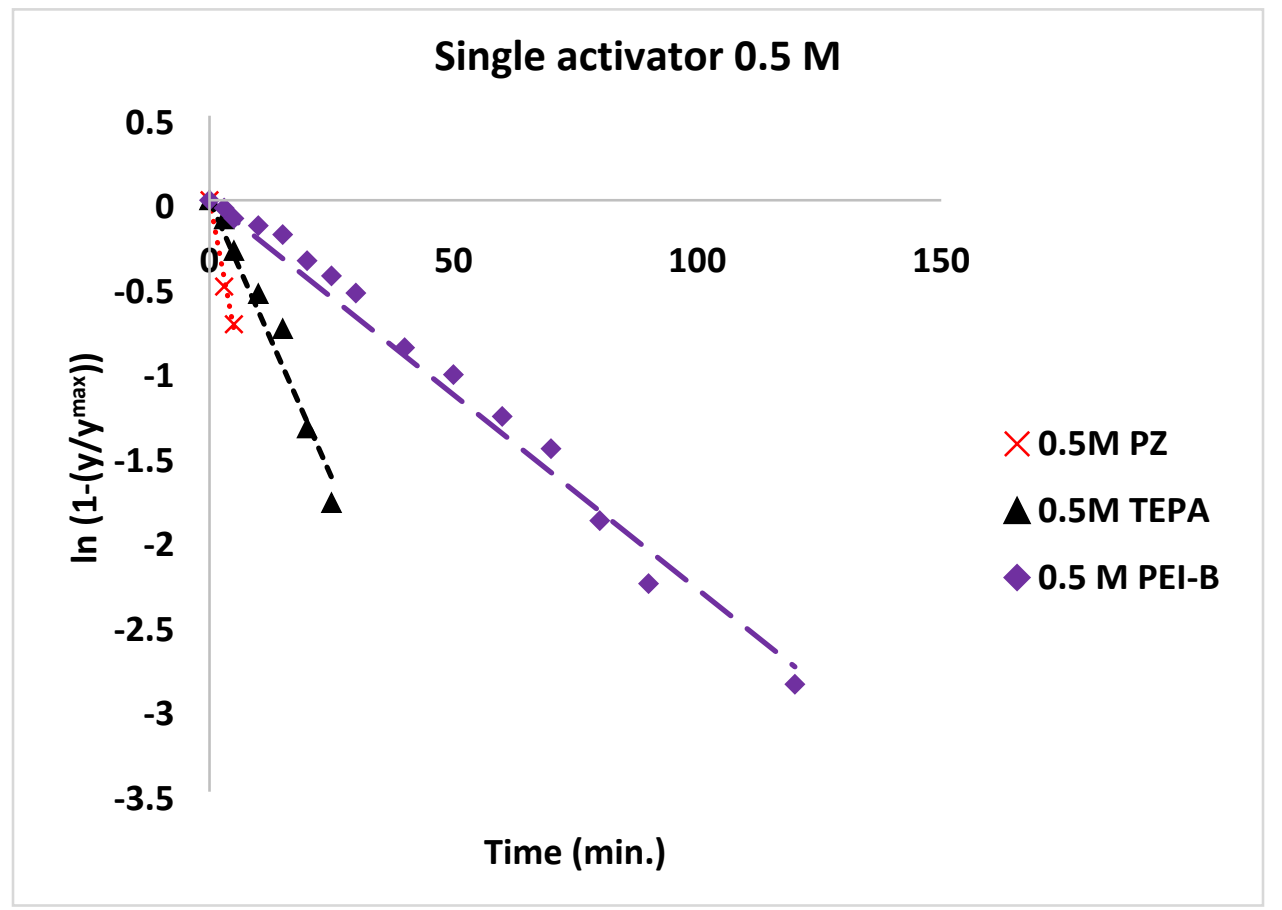

Figure S9. Plot of first order exponential rise expression using $0.5 \mathrm{M}$ polyamine concentration. 


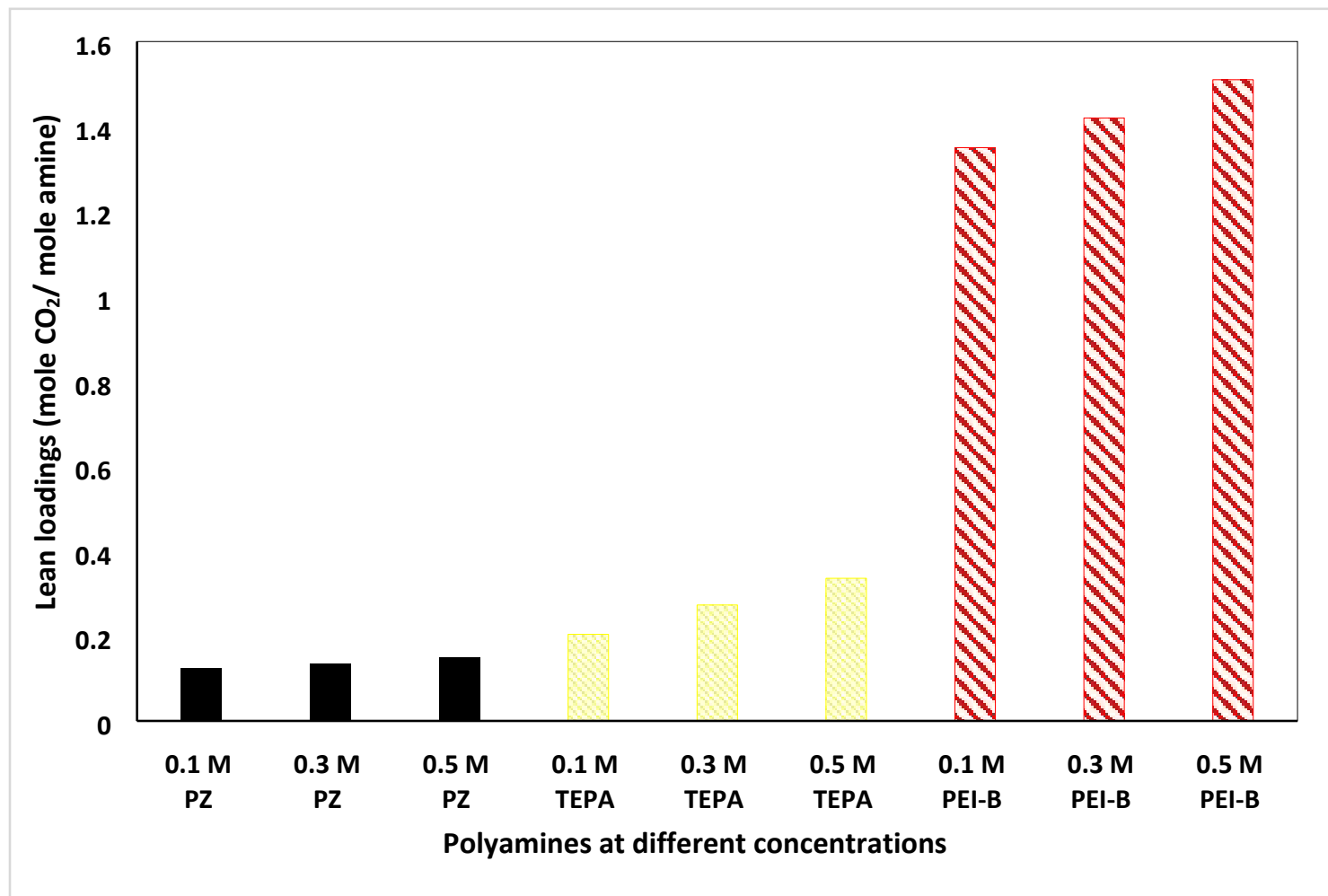

Figure S10. Lean loading values of individual polyamines (PZ, TEPA and PEI-B) using different amine concentrations at $50 \% \mathrm{CO}_{2}$ (balance is $\mathrm{N}_{2}$ ).

\section{$0.1 \mathrm{M}$ polyamine}

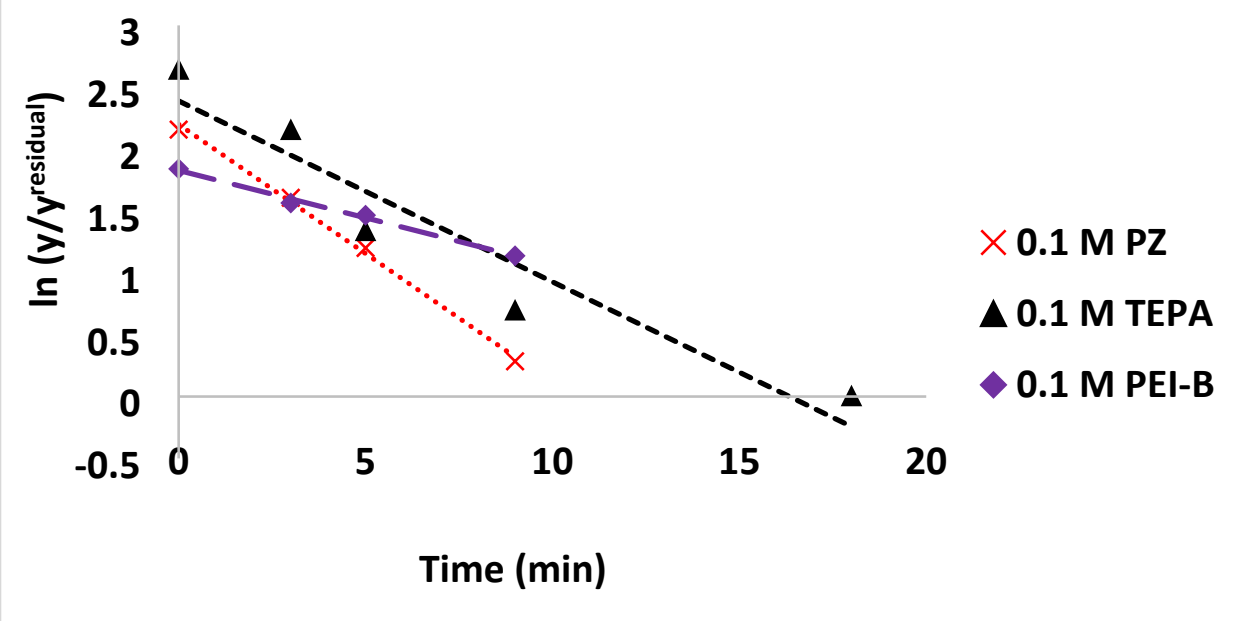


Figure S11. Plot of exponential decay expression using 0.1 M polyamine concentration.

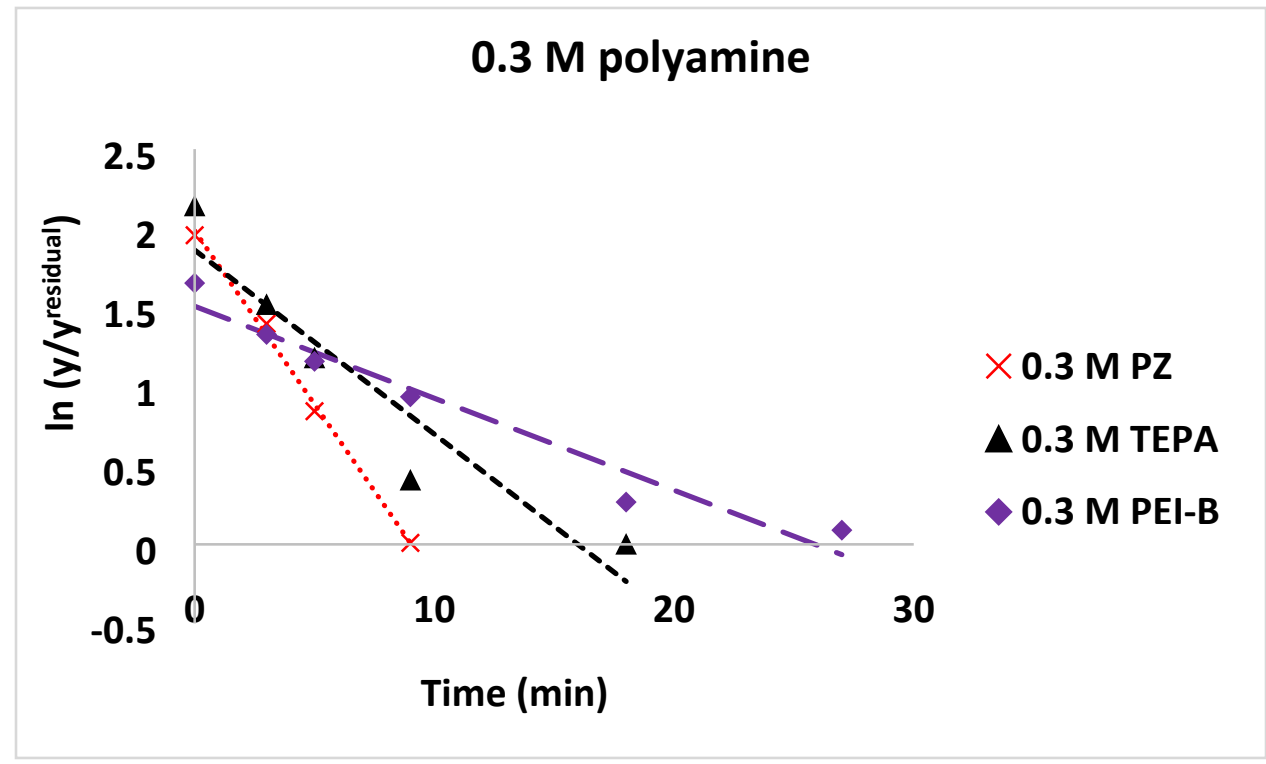

Figure S12. Plot of exponential decay expression using $0.3 \mathrm{M}$ polyamine concentration.

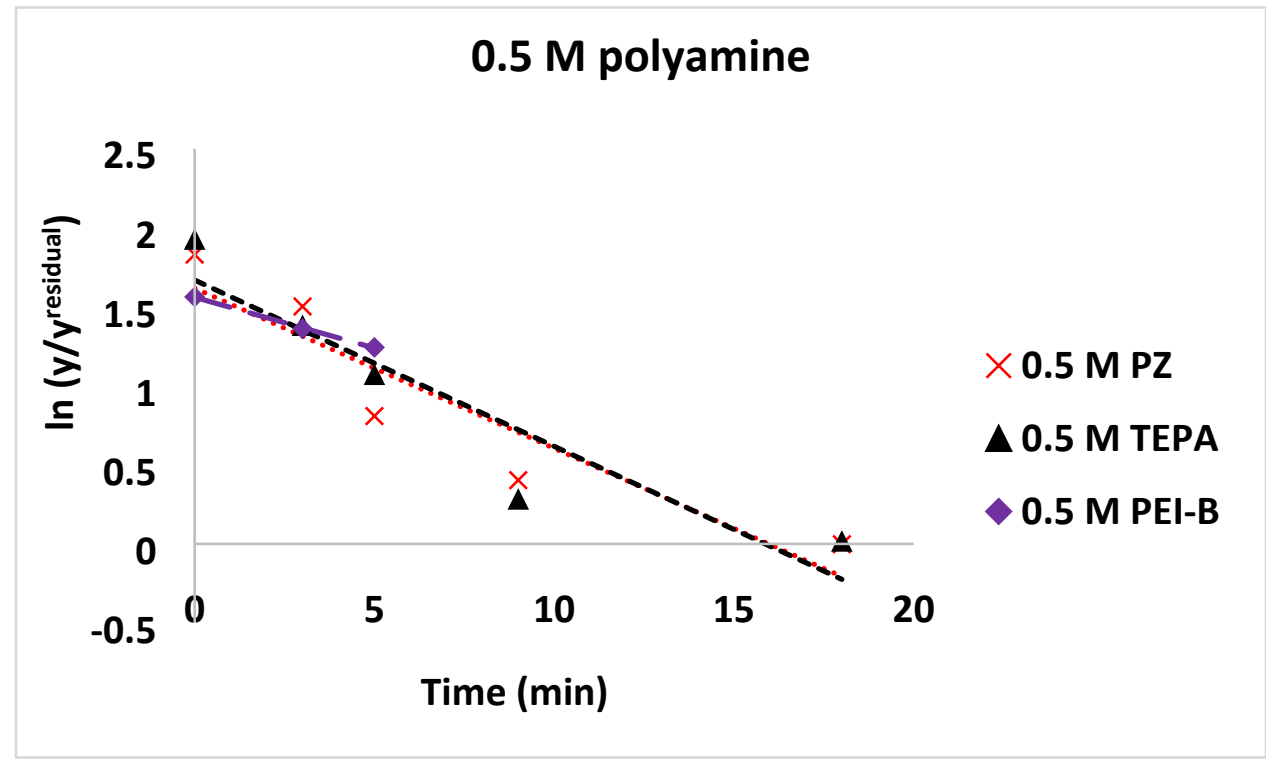

Figure S13. Plot of exponential decay expression using $0.5 \mathrm{M}$ polyamine concentration. 
Table S2. Experimental results of individual polyamines at different amine concentrations using $50 \% \mathrm{CO}_{2}$ (balance is $\mathrm{N}_{2}$ ) by a simple screening setup.

\begin{tabular}{|l|l|l|l|l|l|l|l|r|r|}
\hline Solvent & $\begin{array}{l}0.1 \mathrm{M} \\
\mathrm{PZ}\end{array}$ & $\begin{array}{l}0.3 \mathrm{M} \\
\mathrm{PZ}\end{array}$ & $\begin{array}{l}0.5 \mathrm{M} \\
\mathrm{PZ}\end{array}$ & $\begin{array}{l}0.1 \mathrm{M} \\
\text { TEPA }\end{array}$ & $\begin{array}{l}0.3 \mathrm{M} \\
\text { TEPA }\end{array}$ & $\begin{array}{l}0.5 \mathrm{M} \\
\text { TEPA }\end{array}$ & $\begin{array}{l}0.1 \mathrm{M} \\
\text { PEI-B }\end{array}$ & $\begin{array}{l}0.3 \mathrm{M} \\
\text { PEI-B }\end{array}$ & $\begin{array}{l}0.5 \mathrm{M} \\
\text { PEI-B }\end{array}$ \\
\hline $\begin{array}{l}\text { Rich } \\
\text { loading } \\
\text { (mole } \\
\mathrm{CO}_{2} / \text { mole } \\
\text { amine) }\end{array}$ & 1.06 & 0.95 & 0.93 & 2.86 & 2.32 & 2.29 & 8.5 & 7.41 & 7.2 \\
\hline $\begin{array}{l}\text { Lean } \\
\text { loading } \\
\text { (mole } \\
\mathrm{CO}_{2} / \text { mole } \\
\text { amine) }\end{array}$ & 0.123 & 0.135 & 0.149 & 0.204 & 0.273 & 0.336 & 1.35 & 1.42 & 1.51 \\
\hline $\begin{array}{l}\text { Cyclic } \\
\text { capacity } \\
\text { (mole } \\
\mathrm{CO}_{2} / \mathrm{L} \text { sol) }\end{array}$ & 0.0941 & 0.2457 & 0.3902 & 0.2657 & 0.6132 & 0.9817 & 0.7154 & 1.7987 & 2.8453 \\
\hline $\begin{array}{l}\text { Desorption } \\
\text { rate (mole } \\
\mathrm{CO}_{2} / \text { min L } \\
\text { sol) }\end{array}$ & 0.0098 & 0.027 & 0.0407 & 0.0277 & 0.0617 & 0.1006 & 0.0464 & 0.1239 & 0.2027 \\
\hline $\begin{array}{l}\text { Heat duty } \\
(\mathrm{GJ} / \text { tonne } \\
\mathrm{CO}_{2} \text { ) }\end{array}$ & 302.4 & 109.6 & 72.9 & 126.6 & 56.8 & 34.9 & 86.8 & 32.5 & 19.9 \\
\hline
\end{tabular}




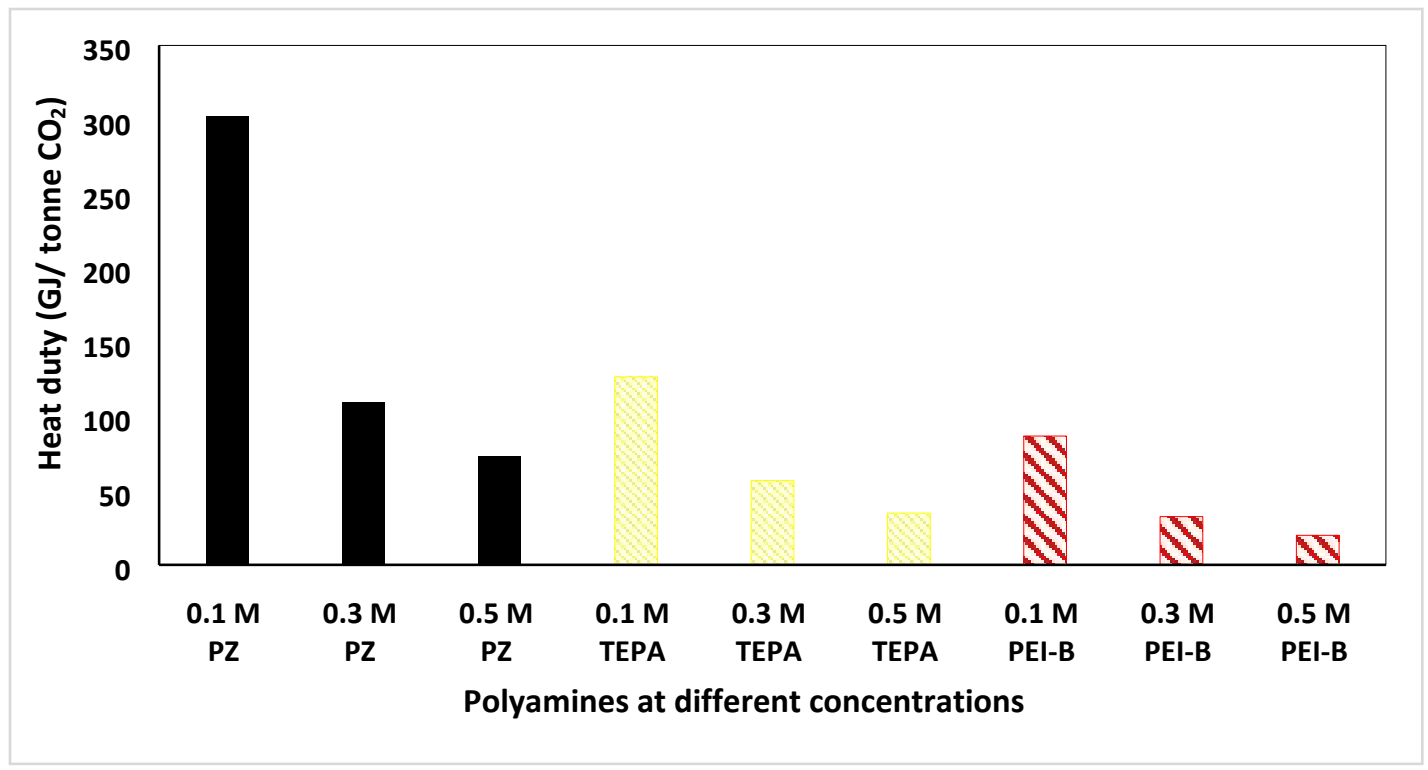

Figure S14. Heat duty values of individual polyamines (PZ, TEPA, and PEI-B) using different amine concentrations at $50 \% \mathrm{CO}_{2}$ (balance is $\mathrm{N}_{2}$ ). 